\title{
Analysis of Antibiotic Resistance Markers in Chlamydia trachomatis Clinical Isolates Obtained after Ineffective Antibiotic Therapy
}

\author{
M. M. Shkarupeta, V. N. Lazarev, T. A. Akopian, \\ T. S. Afrikanova, and V. M. Govorun
}

Translated from Byulleten' Eksperimental'noi Biologii i Meditsiny, Vol. 143, No. 6, pp. 655-659, June, 2007 Original article submitted June 14, 2006

\begin{abstract}
Antibiotic resistance markers were analyzed in C. trachomatis clinical isolates obtained after ineffective therapy of urogenital chlamydiasis with fluoroquinolones and macrolides. Heterotypical resistance to fluoroquinolones and macrolides was detected in all clinical isolates. No significant mutations in the target genes were detected.
\end{abstract}

Key Words: Chlamydia trachomatis; antibiotic resistance markers; heterotypical resistance

Chlamydia trachomatis serovars $\mathrm{D}-\mathrm{K}$ are agents of sexually transmitted infections. Chlamydial infection of the lower compartments of the urogenital tract not detected and treated in time is a direct threat to human reproductive health. Tetracycline, macrolide, and fluoroquinolone antibiotics $(\mathrm{AB})$ are used as etiotropic therapy. However, randomized studies of the efficiency of various protocols of $\mathrm{AB}$ therapy showed that cure rate never reaches $100 \%$ $[8,12]$. Relapses occur in $10-30 \%$ cases $[1,6]$.

The main cause of inefficiency of $A B$ therapy in bacterial infections is drug resistance of the agent. The appearance of $C$. trachomatis clinical isolates resistant to $\mathrm{AB}$ in vitro was reported in many papers $[6,9,13]$. Molecular markers associated with resistance to fluoroquinolones and macrolides were detected in C. trachomatis [2,9]. However, no direct relationship between drug resistance of Chlamydia and efficiency of AB therapy was detected.

Here we studied $\mathrm{AB}$ sensitivity in vitro in cell culture and mutations in the target genes associated

Institute of Physicochemical Medicine, Federal Agency for Health Care and Social Development, Moscow. Address for correspondence: lazar0@newmail.ru. V. N. Lazarev with $\mathrm{AB}$ resistance in $C$. trachomatis clinical isolates obtained after ineffective fluoroquinolone and macrolide therapy.

\section{MATERIALS AND METHODS}

C. trachomatis clinical isolates $(n=9)$ were derived from cervical scrapes in women and urethral scrapes in men. Chlamydia were cultured in McCoy cell cultures with cycloheximide (final concentration $1 \mu \mathrm{g} / \mathrm{ml}$ ). Two $C$. trachomatis reference strains D/UW-3/Cx and E/UW-5/Cx were a kind gift from Dr. Eva Hjelm (Uppsala University, Sweden).

Antibiotic sensitivity was evaluated as described previously [14]. The drug concentration at which none chlamydial incorporations were detected after one passage in McCoy cell culture in medium with $\mathrm{AB}$ was taken for minimum inhibitory concentration (MIC) of this AB. Antibiotic MIC suppressing the formation of typical chlamydial incorporations by at least $90 \%$ was denoted as $\mathrm{MIC}_{90}$. The minimum bactericidal concentration (MBC) was defined as the minimum $\mathrm{AB}$ concentration preventing the formation of incorporations after two passages in cell culture in a medium without $A B$. Levofloxacin and ofloxacin (Hoechst), erythromy- 
cin (Roussel-Ulcaf), roxithromycin and azithromycin (Sigma) were used.

Chlamydial incorporations were detected by the method of direct immunofluorescence in preparations stained with monoclonal antibodies to C. trachomatis outer membrane protein (Orion Diagnostica) using Eclipse E800 fluorescent microscope (Nikon). The morphology and location of chlamydial incorporations in the cells were studied using C1 confocal modulus (Nikon). The images were obtained at 1-sec interval and recorded using EZC1 2.0 Software (Nikon). C. trachomatis DNA was isolated by phenol extraction. The coordinates of C. trachomatis genome and primer areas were determined (Table 1). Amplification parameters were selected in accordance with the thermodynamic characteristics of primers and length of the target fragment. Amplification products were purified using Wizard PCR Preps DNA Purification System (Promega). The amplicon nucleotide sequences were determined on an ABI Prism Genetic Analyzer 3100 sequencer (Applied Biosystems, Hitachi) using Big Dye TM Terminator v.3.0 Cycle Sequencing kit for thermocyclic sequencing (Applied Biosystems). The nucleotide and amino acid sequences of the corresponding gene fragments were analyzed and compared using BLAST and ClustalW software [15]. 3D-simulation of C. trachomatis DNA-gyrase subunit A structure was carried out using FoldRec-CC software on the base of E. coli DNA-gyrase subunit
A structure (Protein Data Bank (http://www.rsgb. org), identification No. lab4).

\section{RESULTS}

Heterotypical sensitivity to $\mathrm{AB}$ was detected in all studied isolates: $A B$ dose suppressing the growth of the bulk of population always differed from the dose completely inhibiting multiplication of the agent (Table 2). The $\mathrm{MIC}_{90}$ value fluctuated in a narrow range and did not surpass the corresponding value for reference strains, while MIC values considerably varied: $0.25-32.00 \mathrm{mg} / \mathrm{liter}$ for levofloxacin, 0.25-128.00 mg/liter for ofloxacin, 0.015-0.500 $\mathrm{mg} / \mathrm{liter}$ for erythromycin, $0.06-2.00 \mathrm{mg} / \mathrm{liter}$ for azithromycin, and 0.03-0.50 mg/liter for roxithromycin. In some clinical isolates MIC significantly surpassed $\mathrm{MIC}_{90}$, which impeded interpretation of the results. For example, judging from $\mathrm{MIC}_{90}$ all clinical isolates could be regarded as fluoroquinolones-sensitive, while according to MIC isolates 257,414 , and 553 could be referred to the group of $\mathrm{AB}$ resistant isolates in comparison with the reference strain (Table 2). MIC of ofloxacin and levofloxacin for isolate 257 was 512- and 16-fold higher than MIC of these drugs from the reference strain, respectively. Similar results were obtained for $\mathrm{MIC}$ and $\mathrm{MIC}_{90}$ for macrolides. Chlamydial growth in the presence of $\mathrm{AB}$ in concentrations below $\mathrm{MIC}_{90}$ differed significantly from the growth in the

TABLE 1. Structure of Primers Used in PCR and Coordinates of Sequences Sites of C. trachomatis Genome

\begin{tabular}{|c|c|c|}
\hline DNA locus & Nucleotide sequence* & Coordinates ${ }^{\star *}$ \\
\hline ompA & $\begin{array}{l}\text { 5'-AGGTTTCGGCGGAGATCC-3' } \\
\text { 5'-ACGTGAGCTGCTCTCTCATCG-3' }\end{array}$ & $778901-779939$ \\
\hline gyrA_1 & $\begin{array}{l}\text { 5'-GGAGACAGAACTACCATGCT-3' } \\
\text { 5'-CCATCCCTACCGCGATGCC-3' }\end{array}$ & $212388-212918$ \\
\hline gyrB_1 & $\begin{array}{l}\text { 5'-CTTCCССTCTCAGATCCCT-3' } \\
\text { 5'-GGTGTTCGTTGCAGCCC-3' }\end{array}$ & $213578-214192$ \\
\hline gyrA_2 & $\begin{array}{l}\text { 5'-ATGCGTCTTACGTCATTTTGG-3' } \\
\text { 5'-TGTAGTCATCCCTACTGCGATG-3' }\end{array}$ & $756031-756452$ \\
\hline gyrB_2 & $\begin{array}{l}\text { 5'-AAGTGGCATCTTTGTGGCGT-3' } \\
\text { 5'-GGCGGAACCCATCTCG-3' }\end{array}$ & $756772-757547$ \\
\hline $\begin{array}{l}\text { 23SrRNA_1 } \\
23 S r R N A 2\end{array}$ & 5'-TATGGTGTGACGCCTGCC-3' & $\begin{array}{l}857 \quad 790-859361 \\
879837-880850\end{array}$ \\
\hline $\begin{array}{l}\text { 23SrRNA_1 } \\
\text { 23SrRNA_2 }\end{array}$ & 5'-ССТССААССТАТСАACCACG-3' & \\
\hline $\begin{array}{l}d m p P \\
t k t B\end{array}$ & $\begin{array}{l}\text { 5'-CGTTATGATCCCAGGATCCCT-3' } \\
\text { 5'-CCCAATATAGAACCGAAAATTCGA-3' }\end{array}$ & $\begin{array}{l}857790-859361 \\
879837-881378\end{array}$ \\
\hline
\end{tabular}

Note. ${ }^{*}$ Identification No. in GenBank of the studied genes nucleotide sequences: NC_000117. ${ }^{* *}$ Numeration for complete sequence of C. trachomatis D/UW-3/Cx genome. 


\begin{tabular}{|c|c|c|}
\hline \multirow{3}{*}{ 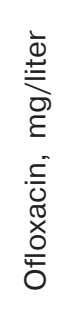 } & $\stackrel{0}{\Sigma}$ & 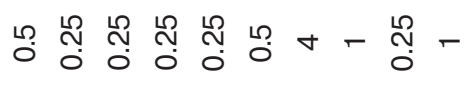 \\
\hline & $\frac{\mathcal{U}}{\Sigma}$ & ָָ̃ \\
\hline & $\stackrel{0}{\infty}^{\circ}$ & 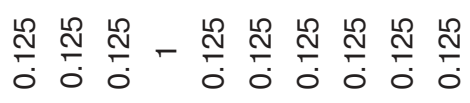 \\
\hline \multirow{3}{*}{ 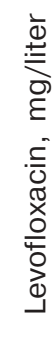 } & $\stackrel{0}{\underline{m}}$ & 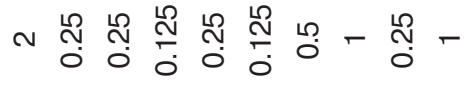 \\
\hline & $\frac{U}{\Sigma}$ & 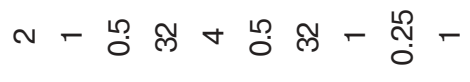 \\
\hline & $\frac{0^{\circ}}{\Sigma}$ & 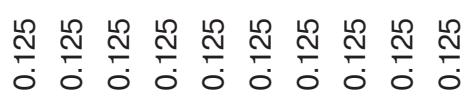 \\
\hline \multirow{3}{*}{ 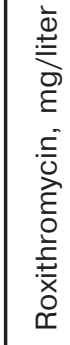 } & $\stackrel{0}{\underline{m}}$ & 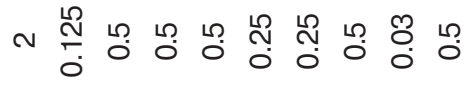 \\
\hline & $\frac{0}{\Sigma}$ & ס̊ \\
\hline & $\frac{0^{\circ}}{\Sigma}$ & 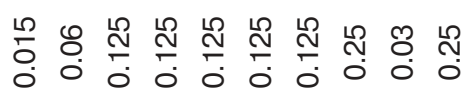 \\
\hline \multirow{3}{*}{ 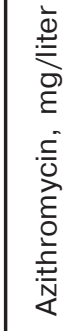 } & $\stackrel{0}{m^{\infty}}$ & 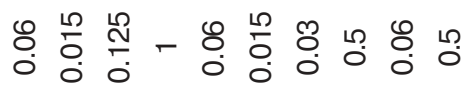 \\
\hline & $\frac{\mathcal{O}}{\Sigma}$ & 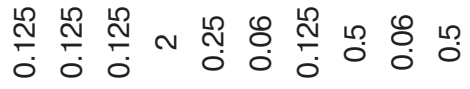 \\
\hline & $\frac{0^{\circ}}{\Sigma}$ & 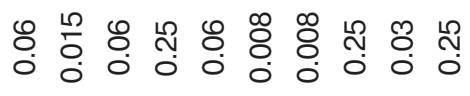 \\
\hline \multirow{3}{*}{ 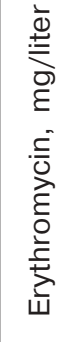 } & $\stackrel{0}{\sum^{\prime}}$ & م્ّ \\
\hline & $\frac{\mathcal{U}}{\Sigma}$ & 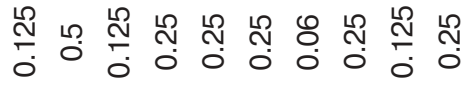 \\
\hline & $\frac{0^{\circ}}{\Sigma}$ & 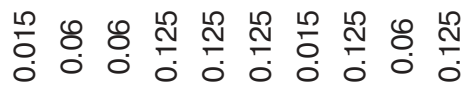 \\
\hline \multicolumn{2}{|c|}{$\begin{array}{l}\stackrel{0}{0} \\
\frac{\pi}{0} \\
\underline{0}\end{array}$} & 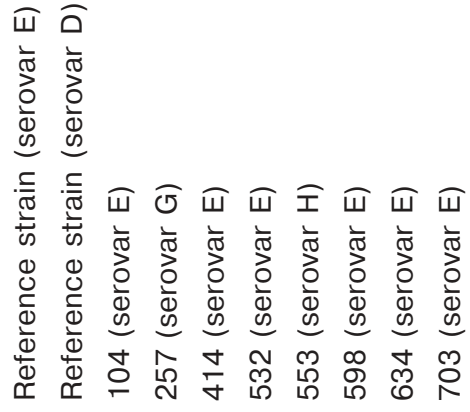 \\
\hline
\end{tabular}

presence of $\mathrm{AB}$ in concentrations ranging from $\mathrm{MIC}_{90}$ to MIC (Fig. 1). In the presence of AB in a concentration below $\mathrm{MIC}_{90}$, the number of infected cells in the monolayer $48 \mathrm{~h}$ after infection was $\geq 50 \%$; typical chlamydial incorporations were well discernible in the cells (large, bulky, shifting the cell nuclei, and containing mature elementary bodies; Fig. 1, $a, d, e, g)$. In contrast, after culturing in the presence of $\mathrm{AB}$ in concentrations from $\mathrm{MIC}_{90}$ to MIC only solitary chlamydial incorporations with atypical morphology were detected (Fig. 1, $b, c, f$, $h)$. MIC was higher than MBC for virtually all isolates, that is, atypical incorporations not always contained mature elementary bodies with infective activity. R. B. Jones et al., who for the first time described a phenomenon when a small part of chlamydial population survived in the presence of high $\mathrm{AB}$ concentrations, called it "heterotypical resistance" [6]. Thus, heterotypical resistance to macrolides and fluoroquinolones was detected in all clinical isolates.

Analysis of QRDR gyrA, gyrB, parC, and parE sequences showed no differences from the structure of analogous sites in the published C. trachomatis sequence (Gen Bank, No. NC_000117) in any of the isolates. Some isolates had 2 mutations in the QRDR neighboring gyrA region: $\mathrm{Val60} \rightarrow$ Ala $(\mathrm{GTA} \rightarrow \mathrm{G} \underline{\mathrm{CA}})$ and $\mathrm{His} 129 \rightarrow \mathrm{Gln} \quad(\mathrm{GA} \underline{\mathrm{C}} \rightarrow \mathrm{GA} \underline{\mathrm{G}})$. Amino acid substitutions in the QRDR region in fluoroquinolone-resistant microorganisms between topoisomerase amino acid residues 67 and 106 were previously detected (substitutions on Ser83 and Asp87 hot points were most incident) [2]. 3D-modeling of C. trachomatis DNA gyrase subunit A showed remote spatial location of amino acid residues 60 and 129 in comparison with location of the known "hot points" (Fig. 2). Mutations were detected only in clinical isolates belonging to genotype $\mathrm{E}$ with different ofloxacin and levofloxacin MIC values; no mutations of this kind were detected in isolates with genotypes $\mathrm{H}$ and $\mathrm{G}$. It is most likely that the identified substitutions are manifestations of gyrA gene polymorphism, specific of genotype E.

No differences were detected in any of genes 23SrRNA_1 and 23SrRNA_2 sites corresponding to 23S rRNA V-domain, in which macrolide resistance-associated mutations are located [9]. Hence, the detected heterotypical fluoroquinolone and macrolide resistance of clinical isolates is not determined by mutations in the target genes associated with $\mathrm{AB}$ resistance.

However, heterotypical AB resistance of Chlamydia is the main cause of ineffective $A B$ therapy of chlamydial infection. Antibiotic therapy is effective, if correct $\mathrm{AB}$ dose is calculated with conside- 

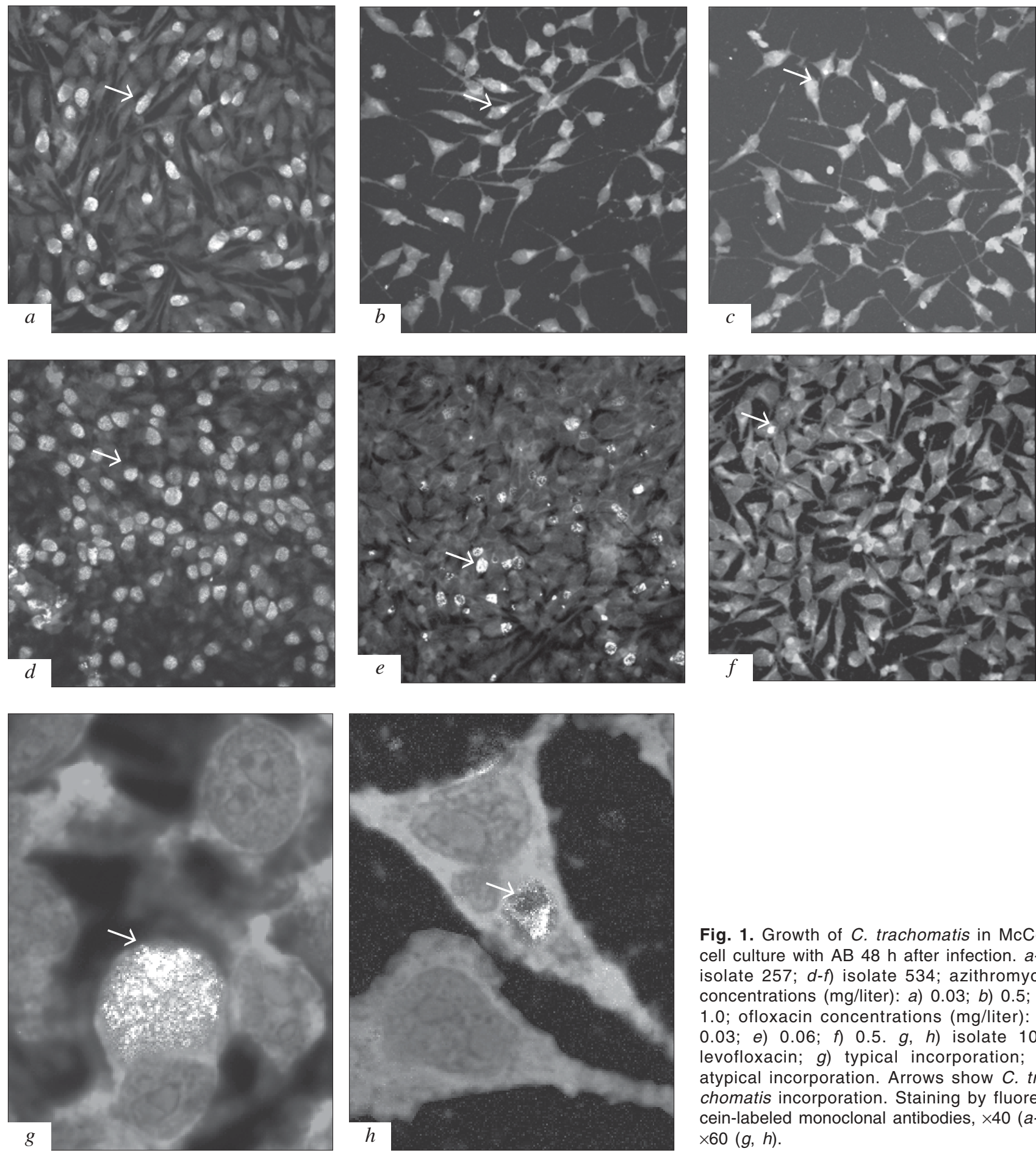

Fig. 1. Growth of C. trachomatis in McCoy cell culture with $A B 48 \mathrm{~h}$ after infection. $a-c$ ) isolate $257 ; d-f$ ) isolate 534 ; azithromycin concentrations (mg/liter): a) 0.03 ; b) 0.5 ; d) 1.0; ofloxacin concentrations (mg/liter): $d$ ) 0.03 ; e) 0.06 ; f) $0.5 . g, h$ ) isolate 104 , levofloxacin; $g$ ) typical incorporation; $h$ ) atypical incorporation. Arrows show C. trachomatis incorporation. Staining by fluorescein-labeled monoclonal antibodies, $\times 40(a-f)$, $\times 60(g, h)$.

ration for MIC. MIC of $\mathrm{AB}$ for obligate intracellular bacteria is a variable depending primarily on the type of cells in which the agent multiplies. This is clearly demonstrated by MIC of azithromycin in vitro [14], which is different for C. trachomatis serovar D in different cell cultures: $1 \mu \mathrm{g} / \mathrm{ml}$ for BGMK, $0.125 \mu \mathrm{g} / \mathrm{ml}$ for McCoy, $0.016 \mu \mathrm{g} / \mathrm{ml}$ for Hela, and $0.008 \mu \mathrm{g} / \mathrm{ml}$ for HL cells.
MIC of $\mathrm{AB}$ directly depends on the time of $\mathrm{AB}$ penetration into the cells (before or after infection). This is confirmed by the results of in vitro experiments: fluoroquinolones in a concentration of 64 $\mu \mathrm{g} / \mathrm{ml}$ do not inhibit the formation of chlamydial bodies if they are added $20 \mathrm{~h}$ after infection [10]; MIC of macrolides, fluoroquinolones, and tetracyclines drastically increase if these $\mathrm{AB}$ are added $8 \mathrm{~h}$ after infection [14]. In turn, according to phar- 


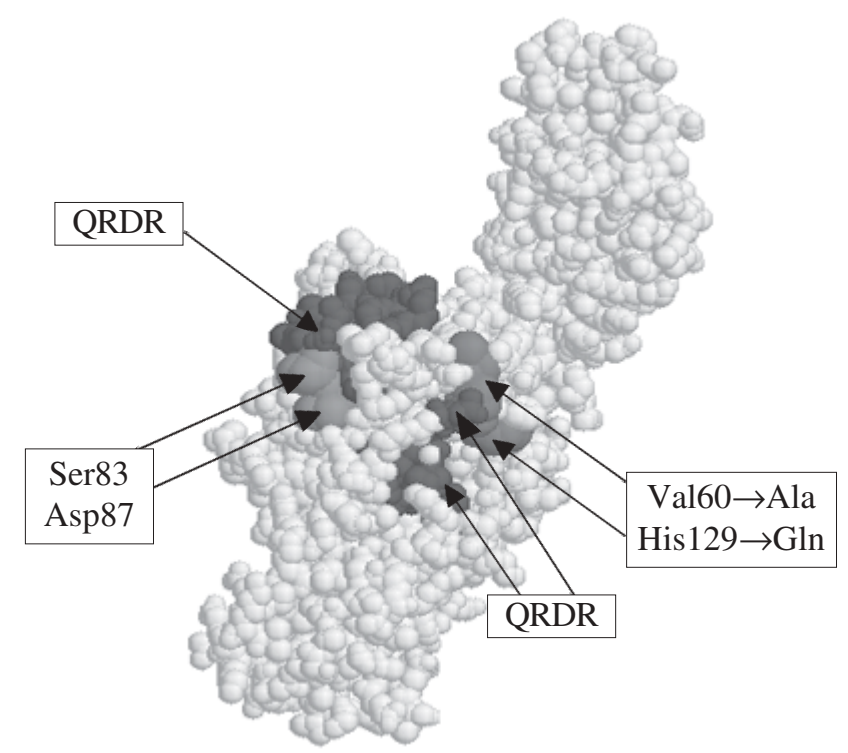

Fig. 2. 3D-model of $C$. trachomatis DNA gyrase subunit A structure.

macokinetic regularities, active concentration of $\mathrm{AB}$ is different in the blood and tissues and changes with time. Heterotypical resistance is an additional factor, contributing to $\mathrm{AB}$ inefficiency, if its concentration is higher than the minimum tolerated concentration, but lower than MIC.

Analysis of growth and morphology of chlamydial inclusions in the presence of $\mathrm{AB}$ suggests that heterotypical resistance is a manifestation of transition of a part of the agent population into a persistent state under the effect of certain $A B$ doses. Persistence of Chlamydia is a part of vital cycle of these microorganisms, it can be easily reproduced in cell culture under the effects of some factors, including $\mathrm{AB}$ [4]. Azithromycin, ciprofloxacin, and ofloxacin are inductors of Chlamydia persistence, they interrupt the productive intracellular cycle of Chlamydia, the microorganisms stop active multiplication, but retain metabolic activity, which is seen from negative result of cultural study and detection of short-living rRNA transcripts $[3,4]$.
Hence, the inefficiency of $A B$ therapy is not caused by the formation of $\mathrm{AB}$ resistant forms of Chlamydia leading to significant mutations in target genes. Inefficiency of $\mathrm{AB}$ therapy can be caused by improperly selected $\mathrm{AB}$ dose and failure of effective control of heterotypically resistanct agent and by transition of the agent into a persistent state under the effect of $\mathrm{AB}$.

The authors are grateful to I. Yu. Torshin for his assistance in the creation of a 3D-model of $C$. trachomatis DNA-gyrase subunit A and to N. V. Nikolaeva for biological material from patients with urogenital chlamydiasis.

\section{REFERENCES}

1. M. J. Blythe, B. P. Katz, B. E. Batteiger, et al., J. Pediatr., 121, No. 3, 487-493 (1992).

2. S. Dessus-Babus, C. M. Bebear, A. Charron, et al., Antimicrob. Agents Chemother., 42, No. 10, 2474-2481 (1998).

3. U. Dreses-Werringloer, I. Padubrin, B. Jurgens-Saathoff, et al., Ibid., 44, No. 12, 3288-3297 (2000).

4. U. Dreses-Werringloer, I. Padubrin, H. Zeidler, and I. Kohler, Ibid., 45, No. 11, 3001-3008 (2001).

5. R. J. Hogan, S. A. Mathews, S. Mukhopadnyay, et al., Infect. Immun., 74, No. 4, 1843-1855 (2004).

6. R. B. Jones, B. Van Der Pol, D. H. Martin, and M. K. Shepard, J. Infect. Dis., 162, No. 6, 1309-1315 (1990).

7. H. O. Kjaer, G. Dimcevski, G. Hoff, et al., Sex. Transm. Infect., 76, No. 3, 169-172 (2000).

8. C. Y. Lau and A. K. Qureshi, Sex. Transm. Dis., 29, No. 9, 497-502 (2002).

9. O. Y. Misyurina, E. V. Chipitsyna, Y. P. Finashutina, et al., Antimicrob. Agents Chemother., 48, No. 4, 1347-1349 (2004).

10. T. Notomi, Y. Ikeda, and A. Nagayama, Chemotherapy, 45, No. 4, 242-248 (1999).

11. R. A. Sayle and E. J. Milner-White, Trends Biochem. Sci., 20, No. 9, 374 (1995).

12. V. Skerk, I. Krhen, M. Lisik, et al., Int. J. Antimicrob. Agents, 24, No. 2, 188-191 (2004).

13. J. Somani, V. B. Bhullar, K. A. Workowski, et al., J. Infect. Dis., 181, No. 4, 1421-1427 (2000).

14. R. J. Suchland, W. M. Geisler, and W. E. Stamm, Antimicrob. Agents Chemother., 47, No. 2, 636-642 (2003).

15. J. D. Thompson, D. G. Higgins, and T. J. Gibson, Nucleic Acids Res., 22, 4673-4680 (1994). 